Revue d'histoire de l'Amérique française

ME REVUE D.HISTOIRE DE L'AMÉRIQUE FRANÇAISE

\title{
Un compagnon blaisois de Samuel Champlain : Jean Ralluau (5 janvier 1576 - après le $1^{\mathrm{er}}$ janvier 1628)
}

\section{Robert Le Blant}

Volume 19, numéro 4, mars 1966

URI : https://id.erudit.org/iderudit/302509ar

DOI : https://doi.org/10.7202/302509ar

Aller au sommaire du numéro

Éditeur(s)

Institut d'histoire de l'Amérique française

ISSN

0035-2357 (imprimé)

1492-1383 (numérique)

Découvrir la revue

Citer cet article

Le Blant, R. (1966). Un compagnon blaisois de Samuel Champlain : Jean Ralluau

(5 janvier 1576 - après le $1^{\mathrm{er}}$ janvier 1628). Revue d'histoire de l'Amérique

française, 19(4), 503-512. https://doi.org/10.7202/302509ar d'utilisation que vous pouvez consulter en ligne. 


\section{UN COMPAGNON BLAISOIS DE SAMUEL CHAMPLAIN : JEAN RALLUAU}

\section{( 5 janvier 1576 - après le 1er janvier 1628)}

Lors de son contrat de mariage, le 11 décembre 1611, Champlain fut assisté seulement par cinq amis, dont Jean Ralluau, mal dénommé Ralleau dans les Voyages publiés sous le nom du grand découvreur.

Fils d'Étienne 1er Ralluau, huissier de la chambre de monseigneur le duc, frère du roi (c'est-à-dire de François de Valois, duc d'Anjou, frère d'Henri III), et de Marguerite Dufour, Jean Ralluau fut baptisé le 5 janvier 1576 en la paroisse SaintSauveur de Blois ${ }^{1}$, ayant comme parrains et marraine, honorable homme Jehan Delachartie, marchand de Blois, Martin Voysin, receveur des Tailles en l'élection de cette ville et Jehanne Rousseau, femme de Bertrand Le Marin. Son père se remaria avant le 1er septembre 1584, date du baptême de son 2ème fils, Etienne II avec Marguerite Cosnier ${ }^{2}$. Celle-ci fut, le 8 octobre 1599, marraine d'Hugues Cosnier, fils de Martin Cosnier, pelletier et de Mathurine Mathieu, ayant pour compères, honorable homme Claude Marceau, marchand et honnête homme Hugues Cosnier, pelletier, valet de chambre du roi ${ }^{3}$. La belle-mère de Jean Ralluau était donc très vraisemblablement proche parente des autres enfants de Martin Cosnier; Marie, baptisée le 28 février 1598, Claude, baptisé le 27 août 1601, Jacques, baptisé le 25 juillet 1602 et Simon, baptisé le 26 janvier 1605, tous dans la même paroisse. Son compère Hugues Cosnier, pelletier et valet

1 Cf. Extraits manuscrits pris sur les registres paroissiaux de Blois avant leur destruction par Troüessart, Baptêmes de Saint-Sauveur, à la disposition des lecteurs à la Bibliothèque de Blois.

2 Cf. Idem.

3 Cf. Idem. 
de chambre du roi, paraît avoir été le grand-père maternel de Nicolas Denys, prétendant à la noblesse comme portant d'azur à 3 lozanges d'argent ou d'or à la barre d'argent au milieu de l'écusson chargé de 3 croix de Jérusalem de sable. Ce remarquable personnage eut de son mariage avec Françoise Jesure, au moins 4 enfants: Marie, épouse de Jacques Denys, Hugues II qui figura probablement avec le titre de sieur de Belleau au No 32 de la liste des Cent-associés de la Cie de la NouvelleFrance ${ }^{4}$, Émilien, admis au nombre des cent gentilhommes de la maison du roi et une fille, Françoise ${ }^{5}$.

Étienne II Ralluau eut pour parrain et marraine noble Étienne Dufour, conseiller et secrétaire du roi, honorable homme Philibert Chéreault, receveur général du Taillon à Caen et Isabelle Dufour, fille de défunt honorable homme Etienne Dufour, huissier de la chambre de Monsieur, frère du roi. Jean Ralluau était praticien, le 1er juin 1597, lorsqu'il figura parmi les parents et amis de la fiancée au contrat de mariage de Catherine Duboys, fille de feu Guillain Duboys, marchand pâtissier à Blois, nièce de Louis Duboys, aussi marchand pâtissier dans la même ville ${ }^{6}$. Secrétaire de Pierre du Gua, il l'accompagna, en 1604, en Acadie, visita personnellement avec Champlain la région du Cap Sable, poussa jusqu'à la rivière SaintJean et revint en France avant le 27 octobre 1604, date à laquelle, logé chez Jean Rémy, marchand fripier, rue Tirechappe, il se rendit en la maison des Petits Carreaux, rue de la Limace, paroisse Saint-Germain-l'Auxerrois, siège de la chambre des marchands pelletiers de Paris. Il fit alors exposer aux honorables hommes, Guillaume Tournemine, Antoine Molé et Macé du Moulin, maîtres pelletiers, gardes de la marchandise de pelleteries à Paris, qu'une sentence rendue la veille par le procureur du roi au Châtelet de Paris ordonnait que Rémy représenterait les peaux de castors et de loutres dont il disait être gardien à la requête des gardes de la pelleterie, contradictoirement entre

\footnotetext{
4 Cf. Liste complétée et rectifiée par nous.

5 Cf. Dossiers Bleus 212, article Cosnier, idem.

${ }^{6}$ Cf. Minutes de Bernard, à la date, étude Girault, XI, aux Archives départementales du Loir et Cher.
} 
ces derniers et Ralluau. Agissant au nom de Rémy, maître Antoine Nouel, maître fondeur, présenta la valeur de huit peaux de castor commun constitué en partie avec des peaux de loutres. Les gardes réclamèrent des peaux de martres du Brésil qui se trouvaient dans la chambre de Rémy, lors de la saisie et que Ralluau déclara lui être étrangères. Il affirma ne pas vouloir vendre le lot assimilé à du castor, mais seulement entendre l'utiliser comme il lui conviendrait, offrant de payer aux gardes les frais de saisie et de visite sous réserve de les réclamer en fin de procès. Finalement, main-levée fut accordée moyennant un versement de trente sous, Ralluau se réservant de demander des dommages et intérêts, notamment pour avoir été contraint à un séjour à Paris qui l'avait empêché d'aller à Rouen chercher un caribou que le roi lui avait commandé de faire amener à Saint-Germain-en-Laye ${ }^{6}$ bis.

Ralluau revint en Acadie, en 1606 et en $1607^{7}$, consacrant, durant la même période, au service de la première Compagnie de Mons une activité partielle pouvant être différente de celle qui lui valut de se trouver engagé, avant le 19 février 1607, comme ayant droit de Me Jacques Bourzu, dans un procès avec Samuel Georges et Jean Macain, jugé au présidial de Rennes, le 24 janvier $1609^{8}$. D'après une déclaration de Pierre du Gua, il fit, en Hollande, pour le compte de ce dernier, aux fins d'empêcher les "Flamands" de venir au Canada, un voyage au cours duquel il fut assisté par Nicolas Bauquemare, marchand de Rouen ${ }^{9}$. Cette mission aurait été effectuée avant le 11 avril 1607, date d'une procuration de Pierre du Gua, en vertu de laquelle Ralluau reçut, le 23 avril, à Rouen, de Jacob Bollé, marchand demeurant en cette ville, neveu de Louis Delbec, marchand demeurant à Amsterdam, 245 livres 14 sols constituant le solde de 491 livres 8 sols, somme pour laquelle le même

6 bis $\mathrm{Cf}$. VII, 66, $\mathrm{N}^{\circ}$ 26, au Minutier central des Archives Nationales; communication de Mme Jurgens, conservateur.

7 Cf. Biggar, The Works of S. Champlain (Toronto, 1928), table.

8 Document perdu, comme les autres décisions de ce présidial.

9 Cf. Interrogatoire de Pierre du Gua par le Maître des Requêtes Robert Aubery, du 2 avril 1612, publié par Charles de Beaurepaire dans la Normandie (1893), 8, $8^{\circ} \mathrm{Z} 11535$, à la Bibliothèque Nationale. 
Ralluau avait vendu à Delbec 4 canons et 2 ancres. Ces armes et ustensiles avaient été enlevés à Tadoussac sur un navire appartenant à de Mons, ayant pour capitaine un certain Fabien, c'est-à-dire, vraisemblablement Fabien de Meriscoiena ${ }^{10}$, par le capitaine Handricq Long, fils de Corneille Long et maître du navire le Lion Blanc ${ }^{11}$. Ralluau fut nanti d'une autre procuration, donnée par de Mons, le 20 octobre 1607, pour faire saisir, d'une part ${ }^{12}, 1280$ castors rapportés de Tadoussac, sans son mandat ou sa permission, par François Gravé, Sieur du Pont, sur le navire dieppois Le Cygne, commandé par le capitaine Pierre Fouconot ${ }^{13}$ et remis à Lucas Legendre, intéressé au navire; d'autre part, 500 castors et loutres traités par le capitaine Morel, le jeune, et son pilote Duglas, à Niganiche, où ils avaient été envoyés par Gravé, Legendre et leurs associés. Ralluau exécuta sa mission, puis donna une main-levée partielle approuvée par son mandat, contribuant ainsi à une solution d'âpaisementit donnt l'inciduence fut loin d'être dénuée d’importance sur les participations à la fondation de Québec.

L'homme d'affaires de Pierre du Gua fut encore pourvu d'une procuration, le 17 octobre 1608, pour faire assigner devant le lieutenant tenant la table de Marbre à Rouen ${ }^{14}$, les marchands malouins Pierre Ribertière, sieur de la Hamelinais, Etienne Goret, sieur du Tertrebarré, Luc Serre, sieur de la Pasquerie, François Martin, sieur de la Vigne et Jean Sarcel, sieur de Prévert, pour avoir contrevenu aux défenses portées à la commission accordée par le roi au lieutenant-général le 7 janvier 1608, en exécution d'une sentence de l'Amirauté de Rouen et de nouvelles lettres royales du 13 octobre. Après avoir encaissé, par l'intermédiaire de Ralluau, le quart des profits de la traite réalisée en 1608, grâce au voyage effectué à Gaspé et à l'Ile Percée sur le navire Le Grand-Saint-Jean-de-Saint-Bris ayant

$10 \mathrm{Cf}$. Revue d'Histoire de l'Amérique française, X, 3: 355.

$11 \mathrm{Cf}$. Tabellionage de Rouen, meubles, 1ère série, 1er janvier au 30 juin 1607, aux archives départementales de la Seine Maritime.

12 Cf. Registre de Cuvillier, XV, 17, fo. 732, aux Archives Nationales.

$13 \mathrm{Cf}$. Main-levée donnée par de Mons, le dernier novembre 1697, minutes d'Antoine Desquatrevaulx, XXIV, à la date.

14 Cf. Cuvillier, XV, 18, fo. 684 . 
comme capitaine Henri Sarcel, comme maître et conducteur Henri Lécuyer, de Mons reconnut, le 30 décembre 1608, avoir passé un accord particulier avec les marchands malouins. Mais Ralluau fut alors remboursé par ces derniers d'une somme de 105 livres avancées par lui pour frais de procédures diligentées aussi par lui en exécution de sa procuration ${ }^{15}$.

De Mons se trouva d'accord, les 3 et 30 octobre 1609, avec Ribertière et Goret pour rétribuer au taux de 600 livres par an les services que Ralluau avait rendus aux associés, de 1604 à 1607. Leur acceptation fut entérinée par des sentences de l'Amirauté de la Table de Marbre à Rouen, des 6, 8 et 13 février 1610. Comme les Malouins agissaient pour le compte de leurs associés, c'est-à-dire les participants malouins et normands, Ralluau ne se trouva plus en conflit qu'avec les rochelais Samuel, Georges et Jean Macain. Les moyens de résistance de ces derniers, brillamment exposés dans un factum déjà publié ${ }^{16}$, furent définitivement anéantis par un arrêt des Maîtres des Requêtes de l'Hôtel du 1er juin $1612^{17}$.

Pendant la seconde Cie de Mons, Ralluau était encore au service de Pierre du Gua qui lui donna une procuration, le 11 décembre 1609, pour réclamer au lieutenant civil du Havre-deGrâce le nègre Mathieu de Coste enlevé par des Hollandais ${ }^{18}$, vraisemblablement à Tadoussac avec les canons et les ancres en 1606. Cet intéressant personnage avait été récupéré et emmené au Canada par Bauquemare qui reconnut, en présence de Ralluau, avoir agi pour le compte du lieutenant-général en NouvelleFrance ${ }^{19}$. Le dévouement de Ralluau lui valut d'être mis en prison à Honfleur avant le 3 mars 1610 sur une procédure de haro intentée par Bauquemare qui réclamait le paiement de ses frais et commença par partager la détention ${ }^{20}$. Mis en liberté,

15 Cf. Idem, fo. 884.

${ }_{16}$ Cf. Morse, Pierre du Gua (Londres, 1939), 73, 74, $4^{\circ} \mathrm{Lm}^{27} 84$ 788 , à la Bibliothèque Nationale.

17 Cf. V4 31, aux Archives Nationales.

18 Cf. Cuvillier, XV, 19, fo. 945.

19 Cf. Idem, fo. 943.

20 Cf. V431, aux Archives Nationales. 
le 3 mars 1610, le malheureux mandataire fit l'objet, le surlendemain, d'un défaut donné aux plaids de Roncheville. Cette juridiction prononça contre lui, le 16 mars, une condamnation qui permit à Bauquemare de faire saisie-arrêt, le 22 mars, sur des sommes dues par Lucas Legendre. Un appel permit à Ralluau d'obtenir une sentence du bailli de Pont-Lévêque en sa faveur, mais son adversaire fit, à son tour, appel devant le parlement de Rouen.

Jean Macain et Samuel Georges n'étaient pas moins tenaces et le parlement de Rennes rendit en faveur de Ralluau, dans l'affaire venue de Bourzu, un arrêt antérieur au 2 juillet 1611, date d'un exécutoire délivré par cette juridiction ${ }^{21}$. Les Rochelais s'acquittèrent, par l'intermédiaire de Mathieu Georges, le 9 décembre 1611, de condamnations prononcées en Bretagne, à concurrence de 542 livres 6 sols 1 denier, et d'une autre résultant d’un exécutoire déiivré par ie présidial de Rennes pour 198 livres 17 sols.

La lutte contre Bauquemare continua de présenter des alternatives diverses. Condamné par arrêt du parlement de Rouen du 20 août $1611^{22}$, Ralluau obtint encore une sentence du bailli de Pont-Lévêque, rendue le 17 janvier 1612 contre le marchand rouennais renvoyé à se pourvoir contre de Mons, mais Bauquemare fit appel devant le parlement de Rouen ${ }^{23}$.

L'arrêt des Maîtres des Requêtes du ler juin 1612, lui ayant donné satisfaction pour ses appointements dus par Pierre du Gua et ses associés, Ralluau trouva moyen de ne pas aller jusqu'au bout dans les procédures d'exécution contre Georges et Macain. Devenu secrétaire du Roi, il habitait rue des Fossés, paroisse Saint-Germain l'Auxerrois et ne s'occupait plus des affaires de Pierre du Gua le 4 août 1612. A cette date, il réussit,

21 Cf. Acte du 11 décembre 1611, registre d'Arragon, LXXXV, 109, aux Archives Nationales.

22 Document perdu.

23 Cf. Arrêt du Parlement de Rouen du 8 juillet 1619, registre du 3 juin au 8 juillet 1619, in fine, aux Archives départementales de la Seine-Maritime. 
par acte passé devant Noël Le Semelier, notaire au Châtelet ${ }^{24}$, à céder à Jacques Le Bodin, sieur de Boisregnard, capitaine du château et du parc de Chambord, demeurant au lieu de Boisregnard, paroisse de Nouy, près de Blois, une créance de 1096 livres, 4 sols, 9 deniers sur Samuel Georges. Il s'agissait de 480 livres représentant la part d'1/5 due par les Rochelais pour ses appointements, de 131 livres 4 sols, somme taxée par les Maîtres des Requêtes pour leurs frais et de 482 livres 9 deniers résultant d'une autre taxe des Maîtres des Requêtes du 2 août 1612 pour frais et dépens. Ces résultats, issus seulement de la dernière décision, attestaient d'une belle persévérance ayant conduit les Rochelais à manger en procès plus que l'objet du litige.

La créance était garantie par une saisie-arrêt effectuée entre les mains de Charles Chelot, marchand, de N., femme Mouschot et François Benoist, marchands, maîtres chapeliers à Paris. Le Bodin à qui Ralluau devait 1000 livres paya les 96 livres 4 sols en espèces, témoignant ainsi une certaine confiance dans l'efficacité de la saisie.

Charles Chelot était, en effet, un important marchand de la rue Saint-Denis, en relations d'affaires, non seulement avec Samuel Georges qu'il avait représenté pour un règlement avec Corneille de Bellois, dès le 31 mars $1607^{25}$, mais aussi avec un autre marchand parisien d'origine auvergnate ${ }^{26}$, Martial Chanut témoin de Champlain lors de son contrat de mariage. François Benoist qui devait être impliqué longtemps dans les affaires du Canada, était chapelier et demeurait rue de la Callandre, paroisse Saint-Germain-le-Vieil, le 30 décembre 1627, lorsqu'il acheta devant Guerreau ${ }^{27}$, la moitié de la part de Pierre Leblond dans la Cie de la Nouvelle-France, pour la revendre, seulement, le 26 février 1638, devant Camuset ${ }^{28}$, à honorable homme Ma-

24 Cf. LIX, 2ème semestre de 1612, 67, aux Archives Nationales.

25 Cf. Tabellionage de Rouen, meubles, 2ème série, 1er janvier-31 mars 1607.

26 Cf. Nouveau Dictionnaire de biographie française, art. Chanut.

$27 \mathrm{Ne}$ figure pas dans LXXXVI, 207.

28 Cf. XXXV, 151, aux Archives Nationales. 
thieu Duisterlo, encore marchand parisien, mais d'origine allemande ${ }^{29}$, un des plus grands spécialistes des affaires portant sur les peaux de castors ${ }^{30}$, au début du XVIIe siècle. Ralluau était secrétaire du commandeur de Sillery, le 24 septembre 1612, lorsqu'il transigea, pour le compte de son oncle, noble homme Robert du Four, sieur de la Corbillière, avec Paul de Saint-Jehan, écuyer, sieur de Segonfiolle, demeurant en la paroisse de la Dralbade, à Toulouse ${ }^{31}$. Il termina ses litiges avec Jean Macain et Samuel Georges par transaction du 25 septembre $1613^{32}$, fut embauché pour soutenir les procès de la Cie du Canada, le 15 novembre suivant ${ }^{33}$, bénéficia ainsi d'une procuration de Samuel Champlain, le 18 mars $1615^{34}$, et représenta, avec le marchand rouennais Daniel Boyer, la Cie du Canada, le 8 août 1617, lors d'un arbitrage passé avec Jacques Pelion, commissaire de Jean Prou et d'autres marchands rochelais ${ }^{35}$, pour soumettre leurs contestations à un arbitrage des maîtres des requêtes conseillers d'État, Amelot, sieur de Chaillon et Regnard qui, finalement, ne fut pas exécuté ${ }^{36}$. Ralluau cumulait les fonctions de secrétaire du commandeur de Sillery et de chargé d'affaires de la Cie du Canada, le 27 novembre 1617, lorsqu'il fut sommé en cette qualité par $\mathrm{Me}$ Guillaume Rangouze, secrétaire du maréchal de Thémines, d'exécuter un accord du 28 novembre 1616 pour l'habitation de Québec et la traite dans la vallée du Saint-Laurent ${ }^{37}$. Ralluau bénéficia, le 9 décembre suivant, d'une cession de Nicolas Delamare, huissier à cheval au Châtelet, demeurant rue Jean Tizon, paroisse Saint-Germain-l'Auxerrois. Le cédant était subrogé pour un capital de 500 livres dans les droits de Jacques de Gaignac, écuyer, sieur de la Couronne, contre Antoine Mercadier, lieutenant de la ville de Limoux

${ }^{29}$ Cf. Minutes de Dournel, XXXV, 75.

$30 \mathrm{Cf}$. Factum pour Nicolas Libert, $4^{\circ} \mathrm{Fm}$ 19503, à la Bib. Nationale.

31 Cf. Minutes de Noel Le Semelier, LIX, 67, déjà citées.

$32 \mathrm{Cf}$. Minutes de Huart, X, 23, aux Archives Nationales.

33 Cf. Anuario de Estudios Americanos, XIV, 1957.

${ }^{34}$ Cf. Revue d'Histoire de l'Amérique française, $\mathrm{X}, 3: 347$.

35 Cf. Registre de Noel Le Semelier, LIX, 74.

36 Cf. Revue d'Histoire de l'Amérique française, X, 3: 347.

37 Cf. Minutes de des Quatreveaulx, XXIV, 250, communiqué par Mme Jurgens, conservateur. 
dans le Bas Languedoc, en exécution d'un arrêt du Grand Conseil ${ }^{38}$. Une rétrocession survint, le 20 septembre $1618^{39}$.

Ralluau obtint définitivement gain de cause contre Bauquemar par un arrêt du parlement de Rouen du 8 juillet 1619 qui ordonna la mainlevée définitive d'une saisie effectuée entre les mains de Lucas Legendre pour une somme de 1000 livres ${ }^{40}$. Il passa une transaction avec un certain Guillaume Barbier, conseiller du roi, le 15 mai $1620,{ }^{41}$ et honorable homme François Costel, marchand, demeurant à Paris, rue Montmartre, paroisse Saint-Eustache, reconnut, le 14 octobre 1627, avoir passé pour son compte, avec noble homme Guillaume Menant, secrétaire du roi, adjudicataire général des Aides de France, la ferme du quart de la ville de Domfront pour 8 ans, moyennent 4500 livres ${ }^{42}$. Qualifié noble homme et secrétaire du roi, Ralluau, qui demeurait alors rue des Mauvaises-Paroles, paroisse SaintGermain-l'Auxerrois, ratifia l'affaire par un autre acte du même jour ${ }^{43}$. Il n'avait ni déménagé, ni modifié ses qualités, le 1er janvier 1628, lorsqu'il consentit en faveur de noble homme $\mathrm{Me}$ Nicolas Rambouillet, secrétaire du roi en sa couronne de Navarre, demeurant rue du Four, paroisse Saint-Eustache, la cession d'une créance de 2000 livres sur les 4000 qu'il avait prêtées pour huit ans à honorable homme Mathieu Pelion, sieur de la Boiaudière, suivant acte du 3 novembre 1627, passé devant Pierre Pellier et Guillaume Bidart, notaires en la vicomté de Domfront ${ }^{44}$.

Le bénéficiaire de l'acte était à la fois l'oncle maternel et le beau-père de l'auteur des célèbres historiettes, Gédéon Tallemant des Réaux, fils de Marie de Rambouillet, sœur de Nicolas et gendre de ce dernier, ayant épousé sa fille Elisabeth. Nicolas Rambouillet était un gros capitaliste de l'époque, brièvement

38 Cf. Minutes de Noël Le Semelier, LIX, 74, fo. 703, ro.

39 Cf. Idem, 75, fo. 403.

40 Cf. Registre des arrêts civils du parlement de Rouen du 3 juin au 8 juillet 1619, in fine, aux Archives de la Seine-Maritime.

41 Cf. Minutes de Noël Le Semelier, 1er semestre 1620, LIX, 54.

42 Cf. Idem, 84, fo. 635.

43 Idem, fo. 636.

44 Cf. Minutes de Jean Le Camus, LIX, 63. 
étudié par Émile Magne ${ }^{45}$, qui a surtout utilisé un embryon de généalogie figurant au Cabinet d'Hozier ${ }^{46}$. C'est très probablement lui qui bénéficia d'une procuration à lui consentie, le 18 août 1622, par Pierre du Gua avec quittance, du 26 décembre suivant ${ }^{47}$, servit de caution au marchand rochelais Samuel Georges et intervint dans les règlements de ce dernier avec les anciens associés de la Cie du Canada, d'après l'arrêt du parlement de Rouen, du 27 février $1631{ }^{18}$.

33 rue de Turin,

ROBERT LE BLANT

Paris VIII', France.

45 Cf. Bourgeois et financiers du XVII siècle. La joyeuse jeunesse de Tallemant des Réaux (Paris, 1921), in 18, $8^{\circ} \operatorname{Ln}^{27} 67652$, à la Bibliothèque Nationale, Idem, La fin troublée de Tallemant des Réaux (Paris, 1922), $8^{\circ} \operatorname{Ln}^{27} 61953$.

46 Cf. Art. Rambouillet, 284, à la Bibliothèque Nationale.

47 Cf. Répertoire des minutes d'Arragon, LXXXV, documents perdus.

$48 \mathrm{Cf}$. Arrêts civils, à la date, aux Archives départementales de la Seine-Maritime. 